\title{
Gigantol inhibits cell proliferation and induces apoptosis by regulating DEK in non-small cell lung cancer
}

\author{
YUXING CAI $^{1}$, YI HAO ${ }^{2}$, HUI XU ${ }^{1}, \mathrm{KAICHEN}^{1}$ and BAOZHONG REN ${ }^{3}$ \\ ${ }^{1}$ Department of Respiratory Medicine, Baoji Center Hospital, Baoji, Shaanxi 721008; \\ ${ }^{2}$ Department of Pediatric Surgery, Baoji Maternal and Child Health Care Hospital, Baoji, Shaanxi 721000; \\ ${ }^{3}$ Department of Respiratory Medicine, Baoji Traditional Chinese Medicine Hospital, Baoji, Shaanxi 721001, P.R. China
}

Received February 7, 2020; Accepted September 18, 2020

DOI: $10.3892 /$ etm.2021.10752

\begin{abstract}
Non-small cell lung cancer (NSCLC) is a common type of cancer, with a mortality of $>80 \%$ worldwide. Gigantol is a bibenzyl compound that displays anticancer activity. The aim of the present study was to determine the biological activity of gigantol in NSCLC and to elucidate the underlying molecular mechanism of its action. The expression of DEK proto-oncogene (DEK) was measured in NSCLC tissues and cell lines by reverse transcription-quantitative PCR (RT-qPCR). The results suggested that DEK levels were significantly increased in NSCLC tissues and cell lines compared with adjacent non-tumor tissues and BEAS-2B normal bronchial epithelial cells, respectively. A549 cells were exposed to a series of gigantol concentrations $(0,25,50$ and $100 \mu \mathrm{M})$ and transfected with DEK small interfering RNA. The results of cell viability measured by MTT assay indicated that gigantol significantly decreased cell viability. Additionally, cell proliferation was assessed by CCK-8 and apoptosis was measured by flow cytometry. In comparison with the control group, gigantol treatment inhibited cell proliferation and promoted apoptosis, whereas DEK knockdown increased gigantol-induced suppression of proliferation and acceleration of apoptosis. Additionally, DEK overexpression reversed gigantol-induced effects on proliferation and apoptosis. Moreover, compared with the control group, gigantol treatment decreased Ki-67 and Bcl-2 expression levels, increased Bax expression levels and inactivated the Wnt/ $\beta$-catenin signaling pathway, as assessed by RT-qPCR and/or western blot. DEK knockdown further increased gigantol-induced effects, but DEK overexpression reversed gigantol-induced effects. To conclude, the results of the present study suggested that gigantol inhibited cell proliferation and induced apoptosis by decreasing Ki-67
\end{abstract}

Correspondence to: Dr Baozhong Ren, Department of Respiratory Medicine, Baoji Traditional Chinese Medicine Hospital, 43 Baofu Road, Baoji, Shaanxi 721001, P.R. China

E-mail: renbaozhong2019@aliyun.com

Key words: non-small cell lung cancer, gigantol, DEK proto-oncogene, cell proliferation, apoptosis and $\mathrm{Bcl}-2$ expression, increasing Bax expression and activating the Wnt/ $\beta$-catenin signaling pathway by regulating DEK. The present study indicated the therapeutic potential of gigantol in patients with NSCLC. In addition, DEK may serve as a novel therapeutic target to enhance the effects of gigantol treatment.

\section{Introduction}

Lung cancer has the highest mortality rate among all types of cancer worldwide (1); 85\% of lung cancer cases are non-small cell lung cancer (NSCLC), which has a 5-year survival rate of $<20 \%$ (2). The majority of patients with NSCLC are diagnosed at advanced stages owing to a lack of effective screening and delayed symptom presentation. Surgery is also not an effective therapeutic strategy (3). With an increased understanding of molecular genetics, directed molecular targeted therapy has proven to be helpful for improving clinical outcomes (4). Despite the identification of several biomarkers for patient diagnosis and prognosis, elucidating additional biomarkers to further improve therapeutic effects is important.

Gigantol is a bibenzyl compound extracted from the Thai orchid, Dendrobium draconis (5). In China, gigantol is an active ingredient commonly extracted from Dendrobium officinale, which is a valuable Chinese herbal medicine (6,7). Plants of the Dendrobium genus have been reported to display immunomodulatory, neuroprotective, antioxidant and antitumor effects (8). Similarly, it has been reported that gigantol has anti-inflammation (9), antioxidant (10), antiplatelet aggregation (11) and anticancer effects (5). Furthermore, gigantol has been indicated to inhibit aldose reductase, which may result in diabetic cataract prevention $(12,13)$. The anticancer activity of gigantol has been previously reported in several types of human cancer, including breast (14), liver (15) and lung (16) cancer. For example, in patients with NSCLC, a previous study revealed that gigantol inhibits metastasis (17). However, the effects of gigantol on cell proliferation and apoptosis, as well as its underlying molecular mechanism of action are not completely understood.

DEK proto-oncogene (DEK) was initially discovered in a subtype of acute myelogenous leukemia, in which the DEK nucleoporin 214 genes were fused (18). The DEK architectural protein is present in the chromatin of multicellular organisms (19). DEK has two functional DNA binding domains: One 
is equivalent to the SAP/scaffold attachment factor box and the other overlaps with a di- or multimerization domain (20). To date, it has been revealed that DEK is involved in the tumorigenesis of human cancer by regulating DNA repair, cell differentiation, senescence and apoptosis (21). A previous study suggested that DEK serves a crucial role in tumor progression and also functions as a prognostic biomarker of NSCLC (22). However, the relationship between gigantol and DEK is not completely understood.

The biological function of gigantol in NSCLC and its underlying molecular mechanism were assessed in the present study. The results indicated that gigantol suppressed proliferation and enhanced apoptosis compared with the control group. Additionally, DEK gene expression was significantly decreased following gigantol treatment compared with the control group, and DEK knockdown enhanced gigantol-induced effects on proliferation and apoptosis. Therefore, gigantol may serve as a useful therapeutic agent and DEK may serve as a target of gigantol in patients with NSCLC.

\section{Materials and methods}

Patients and tissues. A total of 30 paired fresh tumor tissues and adjacent non-tumor tissues $5 \mathrm{~mm}$ away from tumor tissues were collected from patients with NSCLC who underwent surgery at Baoji Traditional Chinese Medicine Hospital (Baoji, China) between January and December 2018. The patients included 18 males and 12 females, with a mean age of 56.77 years (range, 44-70 years). Tissue samples were frozen in liquid nitrogen after resection and subsequently stored at $-80^{\circ} \mathrm{C}$ until further use. Written informed consent was obtained from each participant. The present study was approved by the Ethics Committee of Baoji Traditional Chinese Medicine Hospital (approval reference no. 201801001).

Cell culture. NSCLC cell lines (H1299, HCC827, H1650 and A549) and the human normal bronchial epithelial cell line (BEAS-2B) were purchased from the American Type Culture Collection. Cells were maintained in RPMI-1640 medium (Thermo Fisher Scientific, Inc.) supplemented with 10\% FBS (HyClone; Cytiva), $100 \mathrm{U} / \mathrm{ml}$ penicillin and $100 \mu \mathrm{g} / \mathrm{ml}$ streptomycin (Beyotime Institute of Biotechnology) at $37^{\circ} \mathrm{C}$ with $5 \% \mathrm{CO}_{2}$.

Cell viability. Gigantol (4-[2-(4-Hydroxy-3-methoxyphenyl) ethyl]-2,6-dimethoxy-phenol; purity $\geq 98 \%$ ) was purchased from Chroma Biotechnology Co., Ltd. An MTT assay (Beyotime Institute of Biotechnology) was subsequently performed to detect cell viability. Briefly, cells $\left(2 \times 10^{3}\right.$ cell/well $)$ were seeded into a 96-well plate and exposed to various concentrations of gigantol $(0,25,50$ and $100 \mu \mathrm{M})$ for $24 \mathrm{~h}$ at $37^{\circ} \mathrm{C}$. Subsequently, $10 \mu \mathrm{l}$ MTT was added to each well and incubated at $37^{\circ} \mathrm{C}$ for $4 \mathrm{~h}$. Following incubation, $100 \mu \mathrm{l}$ formazan solving liquid was added to each well until samples were completely dissolved. The optical density of each well was measured at a wavelength of $570 \mathrm{nM}$ using a microplate reader (Bio-Rad Laboratories, Inc.).

Cell transfection. A549 cells were seeded ( $5 \times 10^{4}$ cells/well) into 96-well plates and cultured in complete medium containing a series of gigantol concentrations $(0,25,50$ and $100 \mu \mathrm{M})$ at $37^{\circ} \mathrm{C}$ for $24 \mathrm{~h}$. Following treatment, cells were transfected with 50 nM DEK small interfering (si)RNA-1 (5'-UGUCCUCAU UAAAGAAGAA-3'), 50 nM DEK siRNA-2 (5'-CCUUCU GGCAAACCAUUGCCGAAAU-3'), $50 \mathrm{nM}$ non-targeting siRNA negative control (siRNA-NC; 5'-UUCUCCGAACGU GUCACGUTT-3'), $10 \mu \mathrm{g}$ pcDNA3.1 or $10 \mu \mathrm{g}$ pcDNA3.1-DEK (all from Invitrogen; Thermo Fisher Scientific, Inc.) using Lipofectamine ${ }^{\circledR} 2000$ (Invitrogen, Thermo Fisher Scientific, Inc.). At $48 \mathrm{~h}$ post-transfection, cells were used for subsequent experiments.

Reverse transcription-quantitative PCR (RT-qPCR). Total RNA was extracted from tissue specimens and A549 cells using TRIzol ${ }^{\circledR}$ reagent (Invitrogen; Thermo Fisher Scientific, Inc.). After assessing RNA integrity, total RNA was reverse transcribed into cDNA using the FastKing RT kit (Tiangen Biotech Co., Ltd.). The RT conditions were as follows: $42^{\circ} \mathrm{C}$ for $15 \mathrm{~min}$ and $95^{\circ} \mathrm{C}$ for $3 \mathrm{~min}$ before chilling on ice. Subsequently, qPCR was performed using FastFire qPCR PreMix (SYBR Green; Tiangen Biotech Co., Ltd.) and the ABI PRISM 7500 Real-Time PCR System (Applied Biosystems; Thermo Fisher Scientific, Inc.). The following thermocycling conditions were used for qPCR: Initial denaturation at $95^{\circ} \mathrm{C}$ for $1 \mathrm{~min}$; followed by 40 cycles of denaturation at $95^{\circ} \mathrm{C}$ for 5 $\mathrm{sec}$ and annealing/extension at $60^{\circ} \mathrm{C}$ for $10 \mathrm{sec}$. The following primers were used for qPCR: DEK forward (F), 5'-CCGAGA AAGAACCCGAAATGC-3' and reverse (R), 5'-GTGCCT GGCCTGTTGTAAAGC-3'; Ki-67 F, 5'-ACTGCAGCAGAT GGAACTAGG-3' and R, 5'-AGAACAGTAGCGTGATGT TTGG-3'; Bcl-2 F, 5'-TCATGAAATATGCATCTCACTGG-3' and R, 5'-AAATGCAATCCACTGTCACTCTT-3'; Bax F, 5'-AGGGTTTCATCCAGGATCGAG-3' and R, 5'-CACTCG CTCAGCTTCTTGGT-3'; and GAPDH F, 5'-ATGTCGTGG AGTCTACTGGC-3' and R, 5'-TGACCTTGCCCACAGCCT TG-3'. mRNA expression levels were quantified using the $2^{-\Delta \Delta \mathrm{Cq}}$ method (23) and normalized to the internal reference gene GAPDH.

Western blotting. Total protein was extracted from tissue samples and transfected cells using pre-cooled RIPA lysis buffer (Beyotime Institute of Biotechnology). The BCA Protein Assay kit (Beyotime Institute of Biotechnology) was used to determine the concentration of total protein. Proteins $(30 \mu \mathrm{g})$ were separated via 10\% SDS-PAGE and transferred onto PVDF membranes (Thermo Fisher Scientific, Inc.). After blocking with $5 \%$ skim milk at room temperature for $1 \mathrm{~h}$, the membranes were incubated at $4^{\circ} \mathrm{C}$ overnight with the following primary antibodies (all purchased from Abcam): Anti-DEK (cat. no. ab166624; 1:1,000), anti-Ki-67 (cat. no. ab92742; 1:5,000), anti-Bcl-2 (cat. no. ab182858; 1:2,000), anti-Bax (cat. no. ab53154; 1:1,000) anti-Wnt10b (cat. no. ab70816; $1: 500)$, anti- $\beta$-catenin (cat. no. ab6302; 1:4,000) and anti-GAPDH (cat. no. ab9485; 1:2,500). Following primary incubation, the membranes were incubated with a horseradish peroxidase-conjugated goat anti-rabbit IgG secondary antibody (cat. no. ab205718; 1:2,000; Abcam) for $1 \mathrm{~h}$ at room temperature. Protein bands were visualized using the EasyBlot ECL kit (Sangon Biotech, Co., Ltd.). Protein expression levels were semi-quantified using Image J software (version 1.48U; 

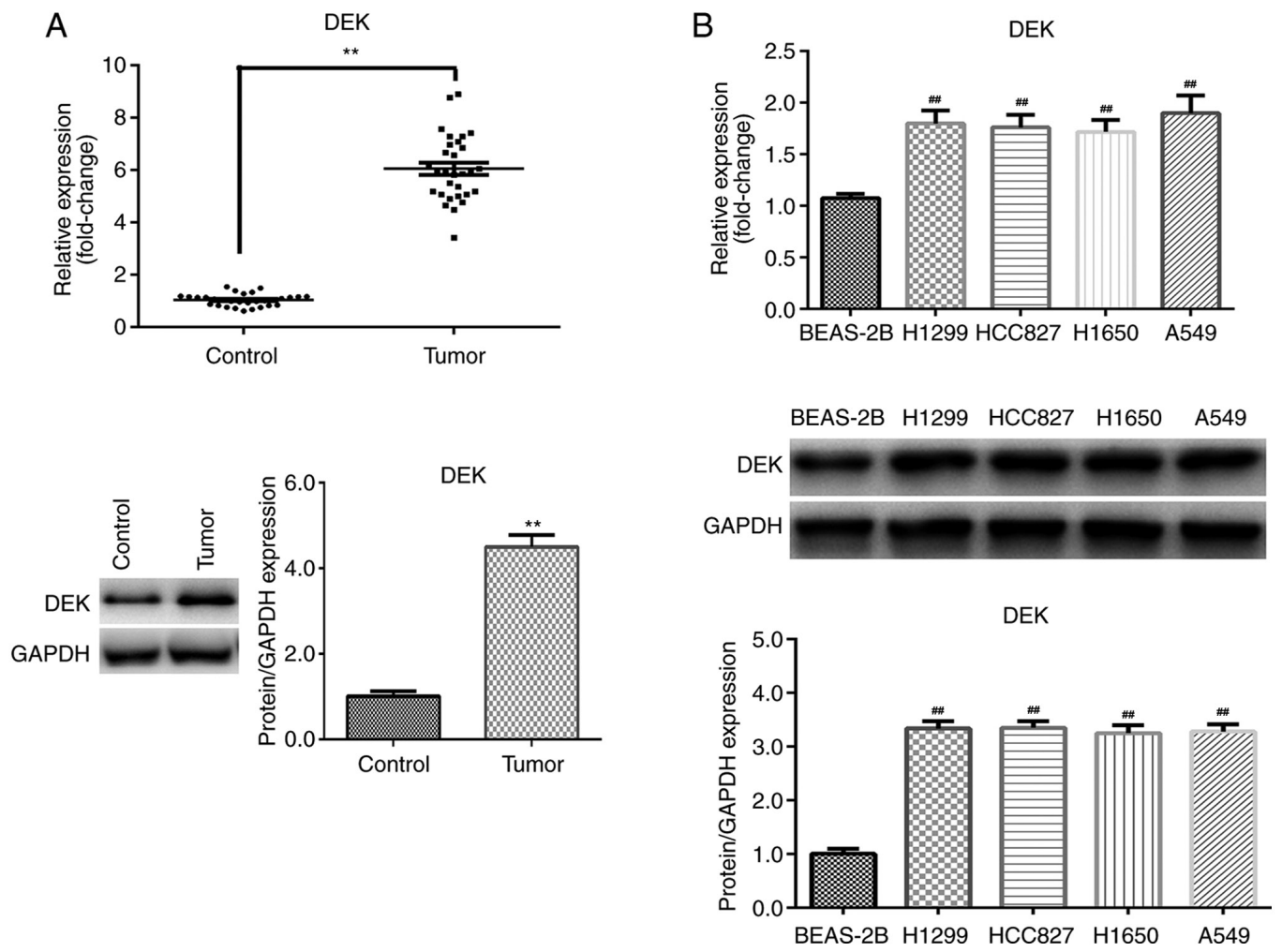

Figure 1. DEK expression is upregulated in NSCLC tissues and cells. (A) DEK mRNA and protein expression levels were measured in 30 paired tumor and adjacent non-tumor tissues by RT-qPCR and western blotting, respectively. ${ }^{* *} \mathrm{P}<0.01$ vs. control. (B) DEK mRNA and protein expression levels were also measured in NSCLC cell lines (A549, H1299, HCC827 and H1650) and a normal bronchial epithelial cell line (BEAS-2B) by RT-qPCR and western blotting, respectively. Data are presented as the mean \pm SEM. ${ }^{\# \#} \mathrm{P}<0.01$ vs. BEAS-2B. DEK, DEK proto-oncogene; NSCLC, non-small cell lung cancer; RT-qPCR, reverse transcription-quantitative PCR.

National Institutes of Health) with GAPDH as the loading control.

Cell proliferation assay. Cell proliferation was assessed using the Cell Counting Kit-8 (CCK-8) assay (MedChemExpress) following the manufacturer's protocol. Cells were seeded $\left(1 \times 10^{4}\right.$ cells/well) into 96 -well plates and incubated at $37^{\circ} \mathrm{C}$ with $5 \% \mathrm{CO}_{2}$. for $0,12,24$ and $48 \mathrm{~h}$. Subsequently, CCK-8 reagent $(10 \mu \mathrm{l})$ was added to each well at $37^{\circ} \mathrm{C}$ with $5 \% \mathrm{CO}_{2}$ for $4 \mathrm{~h}$. Absorbance was measured at a wavelength of $450 \mathrm{nM}$ using a microplate reader (Bio-Rad Laboratories, Inc.).

Apoptosis assay. Apoptosis was assessed using the Annexin V Apoptosis Detection Kit FITC (Sangon Biotech Co., Ltd.). Transfected cells were resuspended in binding buffer at a density of $2 \times 10^{5}$ cells $/ \mathrm{ml}$. Following incubation with $5 \mu \mathrm{l}$ Annexin V-FITC and $10 \mu 1$ PI for 15 min at room temperature in the dark, flow cytometry was performed. Early and late apoptotic cells were analyzed using the CytoFLEX flow cytometer (Beckman Coulter, Inc.) carrying CellQuest Pro software (version 5.1; BD Biosciences).

Statistical analysis. Each experiment was repeated at least three times. Statistical analyses were performed using GraphPad Prism software (version 6.0; GraphPad Software, Inc.). Data are presented as the mean \pm SEM. Comparisons between two unrelated groups were analyzed using unpaired Student's t-test. However, comparisons between tumor tissues and adjacent normal tissues used paired Student's

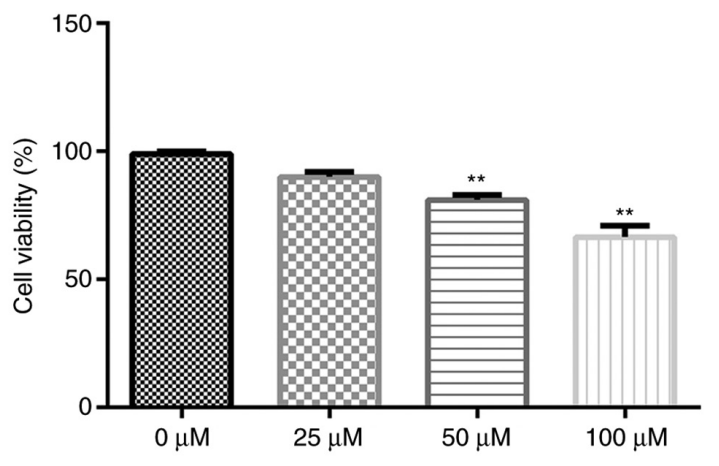

Figure 2. Effect of gigantol on cell cytotoxicity. A549 cells were exposed to various concentrations of gigantol $(0,25,50$ and $100 \mu \mathrm{M})$ for $24 \mathrm{~h}$. Subsequently, cell viability was determined by performing an MTT assay. Data are presented as the mean $\pm \mathrm{SEM}$. ${ }^{* *} \mathrm{P}<0.01$ vs. $0 \mu \mathrm{M}$.

t-test. Comparisons among multiple groups were analyzed using one-way ANOVA followed by Tukey's post hoc test. $\mathrm{P}<0.05$ was considered to indicate a statistically significant difference.

\section{Results}

DEK expression levels are increased in NSCLC. To determine the function of DEK in NSCLC, mRNA and protein expression levels in tissues and cells were first assessed by RT-qPCR and western blotting, respectively. The results indicated that DEK expression was significantly upregulated in tumor 
A

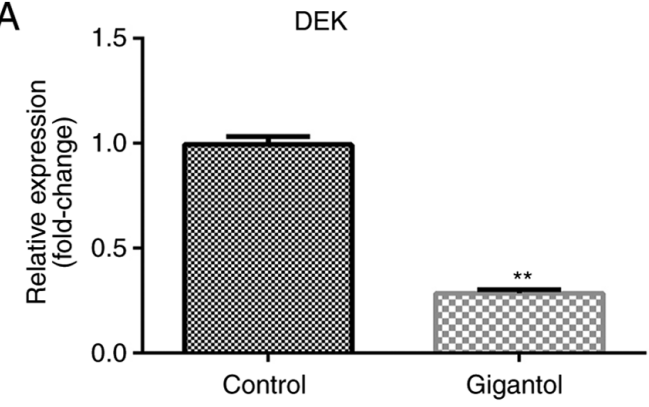

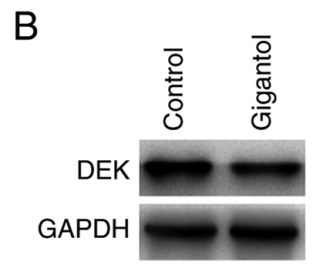

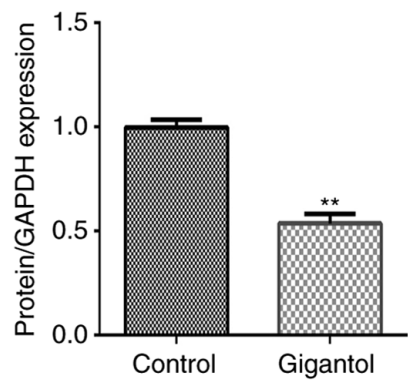

Figure 3. Gigantol decreases DEK expression levels in A549 non-small cell lung cancer cells. (A) DEK mRNA and (B) DEK protein expression levels were detected measured by reverse transcription-quantitative PCR and western blotting, respectively. Data are presented as the mean \pm SEM. ${ }^{* *} \mathrm{P}<0.01$ vs. control. DEK, DEK proto-oncogene.

tissues compared with the paired adjacent non-tumor tissue control group $(\mathrm{P}<0.01$; Fig. 1A). Additionally, compared with BEAS-2B cells, DEK expression levels were significantly increased in NSCLC cell lines $(\mathrm{P}<0.01$; Fig. 1B), most notably in A549 cells. Therefore, A549 cells were used in subsequent experiments.

Cytotoxicity of gigantol in A549 cells. To determine the cytotoxicity of gigantol, A549 cells were exposed to a series of gigantol concentrations $(0,25,50$ and $100 \mu \mathrm{M})$. Subsequently, cell viability was analyzed by performing an MTT assay. The results indicated that gigantol decreased cell viability in a dose-dependent manner (Fig. 2). Compared with the $0 \mu \mathrm{M}$ group, only 50 and $100 \mu \mathrm{M}$ gigantol significantly decreased cell viability $(\mathrm{P}<0.01)$. However, there was no significant difference between the 0 and $25 \mu \mathrm{M}$ groups. Therefore, the non-toxic concentration of $25 \mu \mathrm{M}$ was used for further experiments.

Gigantol regulates DEK expression in A549 cells. To investigate whether gigantol influenced DEK, the expression of DEK was assessed in gigantol-treated cells. The results suggested that DEK mRNA and protein expression levels were significantly decreased by gigantol treatment compared with the untreated control group $(\mathrm{P}<0.01$; Fig. 3A and $\mathrm{B}$, respectively).

DEK knockdown enhances gigantol-mediated inhibition of proliferation and increases gigantol-induced apoptosis. To evaluate the role of DEK in gigantol-treated cells, A549 cells were transfected with DEK siRNA. The transfection efficiency of two DEK siRNAs were determined, which demonstrated that DEK mRNA and protein expression levels were significantly downregulated by DEK knockdown compared with the siRNA-NC group (both $\mathrm{P}<0.01$; Fig. 4A and B), particularly in the DEK siRNA-2 group. Cell proliferation and apoptosis were subsequently analyzed by performing CCK-8 and flow cytometry assays, respectively. Compared with the control group, cell proliferation was significantly inhibited by gigantol $(\mathrm{P}<0.01)$ and further suppressed by DEK knockdown $(\mathrm{P}<0.01$; Fig. 4C). Moreover, apoptosis was significantly increased in the gigantol group compared with the control group $(\mathrm{P}<0.01)$. Gigantol-induced apoptosis was not affected by siRNA-NC transfection, but was significantly increased following DEK siRNA-2 transfection $(\mathrm{P}<0.01$; Fig. 4D and $\mathrm{E})$.

DEK overexpression promotes cell proliferation and suppresses apoptosis in gigantol-treated cells. A549 cells were transfected with pcDNA3.1-DEK overexpression vector and transfection efficiency was measured by RT-qPCR and western blotting. The results indicated that DEK mRNA and protein expression levels were significantly upregulated by pcDNA3.1-DEK compared with pcDNA3.1 empty vector transfection $(\mathrm{P}<0.01$; Fig. $5 \mathrm{~A}$ and $\mathrm{B})$. Moreover, compared with the gigantol + pcDNA3.1 group, DEK overexpression significantly enhanced cell proliferation in gigantol-treated cells ( $\mathrm{P}<0.01$; Fig. 5C). By contrast, gigantol-induced apoptosis was decreased by DEK overexpression, compared with pcDNA3.1 ( $\mathrm{P}<0.01$; Fig. 5D and $\mathrm{E})$.

Gigantol suppresses Ki-67 and Bcl-2 expression, increases Bax expression and inactivates the Wnt/ $\beta$-catenin signaling pathway by regulating DEK. The expression levels of Ki-67 (a proliferation marker) and apoptosis-related factors Bcl-2 and Bax were assessed. Compared with the control group, gigantol significantly reduced $\mathrm{Ki}-67$ and $\mathrm{Bcl}-2$ mRNA expression levels, but increased Bax mRNA expression levels (all $\mathrm{P}<0.01$; Fig. 6A-C, respectively). DEK knockdown enhanced the effects of gigantol on the expression levels of Ki-67, Bcl-2 and Bax $(\mathrm{P}<0.01)$. Similar effects were observed for $\mathrm{Ki}-67, \mathrm{Bcl}-2$ and Bax protein expression levels $(\mathrm{P}<0.01$, Fig. $6 \mathrm{D}$ and $\mathrm{E})$. In addition, the expression levels of Wnt10b and $\beta$-catenin were significantly decreased following gigantol treatment compared with the control group $(\mathrm{P}<0.01)$, and DEK knockdown increased gigantol-mediated downregulation $(\mathrm{P}<0.01$; Fig. 6D and $\mathrm{E})$.

DEK overexpression promotes gigantol-mediated effects on increasing $\mathrm{Ki}-67$ and Bcl-2 expression levels, decreasing Bax expression levels and activating the Wnt/ $\beta$-catenin signaling pathway. RT-qPCR and western blotting results indicated that the mRNA and protein expression levels of $\mathrm{Ki}-67$ and $\mathrm{Bcl}-2$ were significantly increased $(\mathrm{P}<0.01)$, but Bax expression levels were significantly decreased $(\mathrm{P}<0.01)$ by pcDNA3.1-DEK in gigantol-treated A549 cells (Fig. 7A-E). Additionally, gigantol-induced protein expression levels of Wnt10b and $\beta$-catenin were significantly increased by DEK overexpression $(\mathrm{P}<0.01$; Fig. 7D and $\mathrm{E})$. 
A

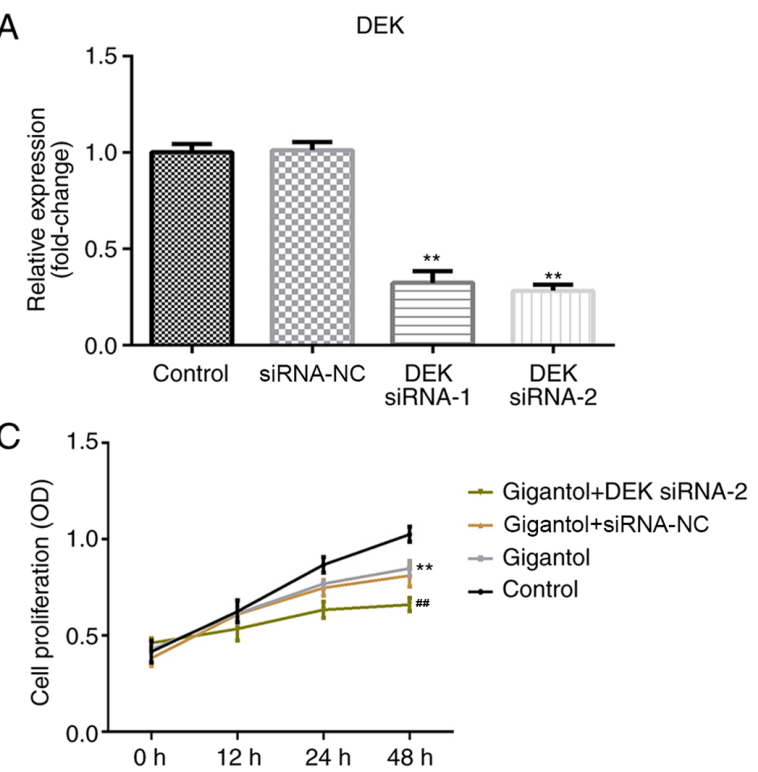

D

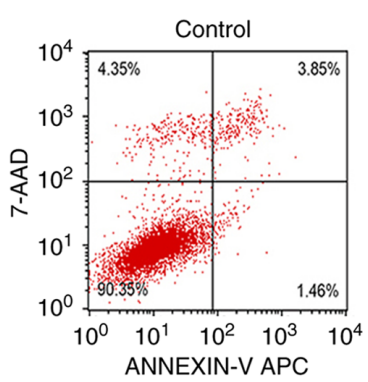

Gigantol+siRNA-NC

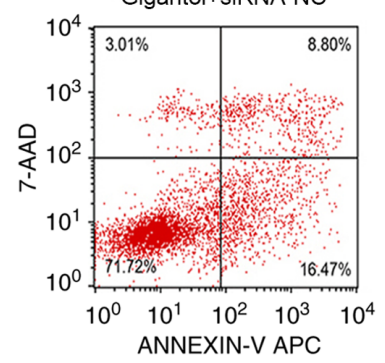

B
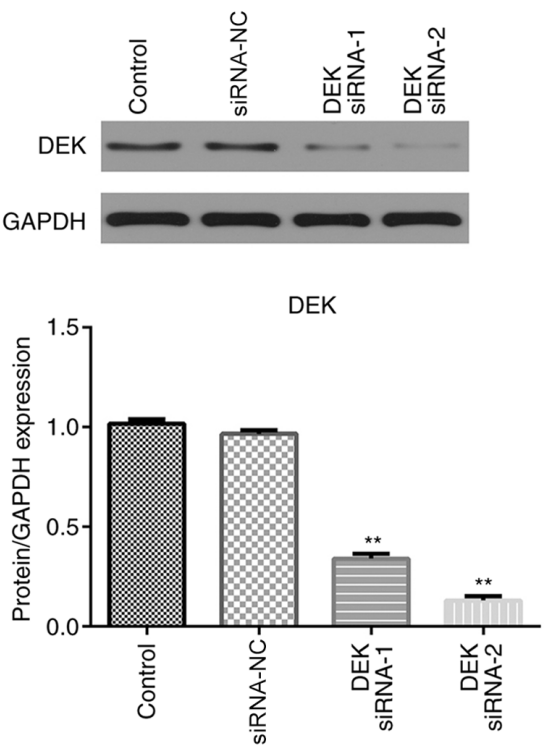

$\mathrm{E}$

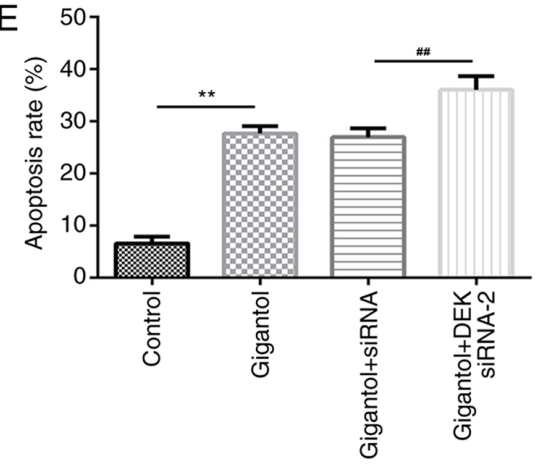

Figure 4. Gigantol inhibits cell proliferation and may promotes apoptosis by regulating DEK. Following transfection with siRNA-NC, DEK siRNA-1 and DEK siRNA-2, transfection efficiency was detected by (A) reverse transcription-quantitative PCR and (B) western blotting. A459 cells were transfected with DEK siRNA-2 and co-treated gigantol for $0,12,24$ and $48 \mathrm{~h} .{ }^{* *} \mathrm{P}<0.01 \mathrm{vs}$. siRNA-NC. Subsequently, (C) cell proliferation was determined by performing a Cell Counting Kit-8 assay. ${ }^{* *} \mathrm{P}<0.01$ vs. control. (D) Flow cytometry was performed to evaluate apoptosis. (E) The rate of apoptosis was quantified. Data are presented as the mean \pm SEM. ${ }^{* *} \mathrm{P}<0.01$ and ${ }^{\# \#} \mathrm{P}<0.01$. DEK, DEK proto-oncogene; OD, optical density; siRNA, small interfering RNA.

\section{Discussion}

Results from the present study demonstrated that DEK expression levels were increased in NSCLC tissues and cells compared with adjacent non-tumor tissues and BEAS-2B cells, respectively. Gigantol treatment inhibited NSCLC cell proliferation and induced apoptosis compared with the control. Furthermore, DEK knockdown enhanced gigantol-mediated inhibition of cell proliferation and promotion of apoptosis. Therefore, gigantol may serve as a potential therapeutic agent through regulating DEK expression for the trepary of NSCLC.

DEK has been identified as an oncogene that regulates cellular processes in many types of cancer. For example, decreased DEK expression inhibited cell proliferation, induced apoptosis and blocked the cell cycle at the $\mathrm{G}_{0} / \mathrm{G}_{1}$ phase in astrocytic tumors (24). Furthermore, DEK overexpression induces the epithelial-mesenchymal transition (EMT) process in osteosarcoma cells (25). Additionally, miR-1292-5p inhibits gastric carcinoma cell proliferation, migration and invasion through suppressing DEK, suggesting DEK is an oncogene (26). DEK is also a prognosis factor in gastric cancer (27), colorectal cancer (28) and NSCLC (22). Moreover, in lung cancer, DEK was highly expressed in tumor tissues, which promoted cell proliferation and invasion (29). Results from the present study demonstrated that DEK expression levels were increased in NSCLC tissues and cells compared with adjacent non-tumor tissues and BEAS-2B cells, respectively, which was consistent with previous studies $(22,29)$. 


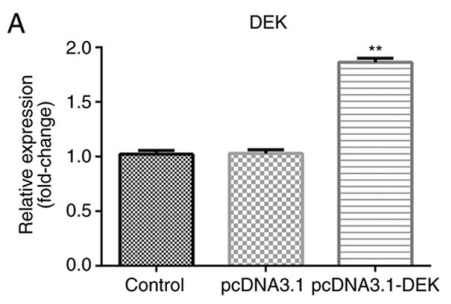

B

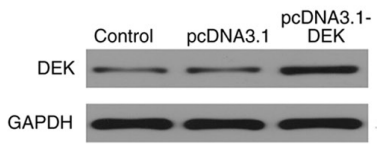

DEK

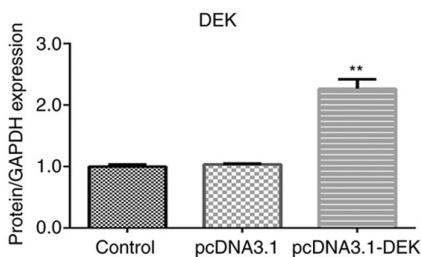

$\mathrm{C}$

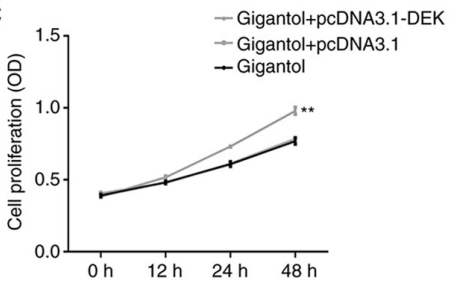

$\mathrm{D}$

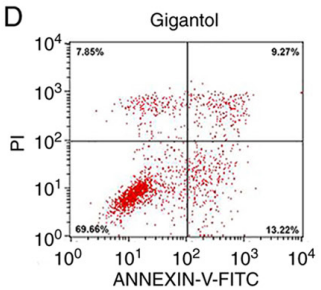

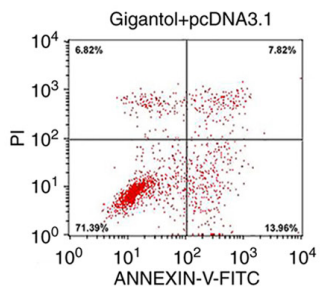
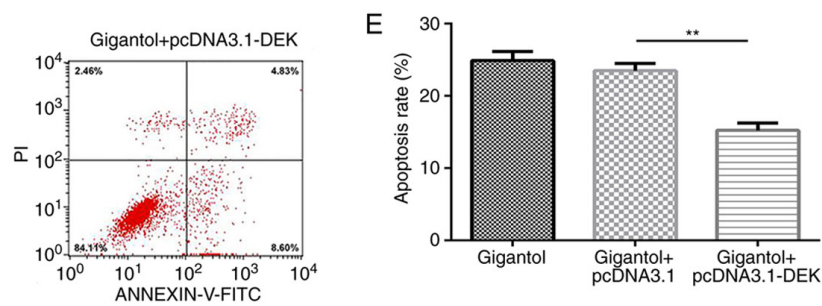

Figure 5. DEK overexpression decreases gigantol-mediated inhibition of cell proliferation and induction of apoptosis. Following transfection with pcDNA3.1 or pcDNA3.1-DEK, transfection efficiency was measured by (A) reverse transcription-quantitative and (B) western blotting. ${ }^{* *} \mathrm{P}<0.01 \mathrm{vs}$. pcDNA3.1. Transfected cells were cultured with gigantol for 0, 12, 24 and 48 h. (C) Subsequently, cell proliferation was assessed by performing a Cell Counting Kit- 8 assay. ${ }^{* *} \mathrm{P}<0.01$ vs. gigantol + pcDNA3.1. (D) Flow cytometry was performed to evaluate apoptosis. (E) The rate of apoptosis was quantified. Data are presented as the mean \pm SEM. ${ }^{* *} \mathrm{P}<0.01$. DEK, DEK proto-oncogene; OD, optical density.

A

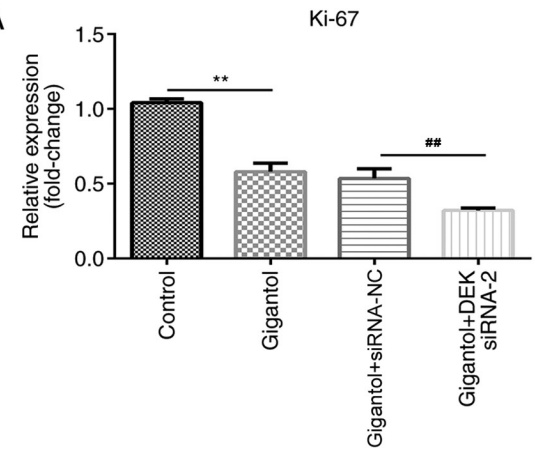

C

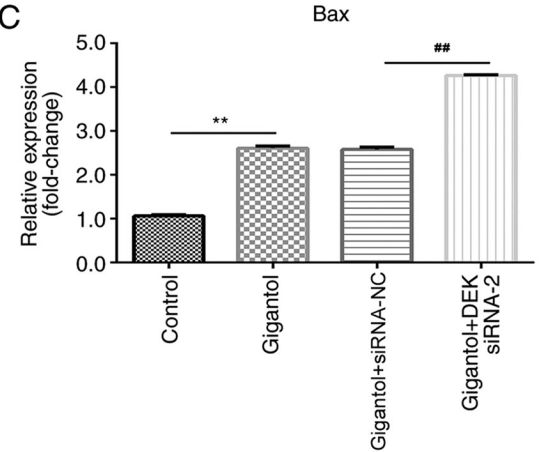

B

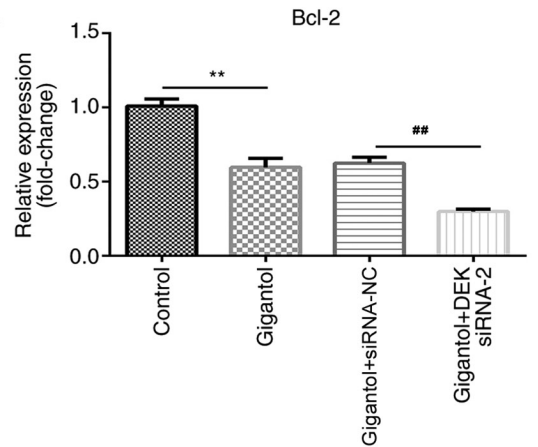

$\mathrm{D}$

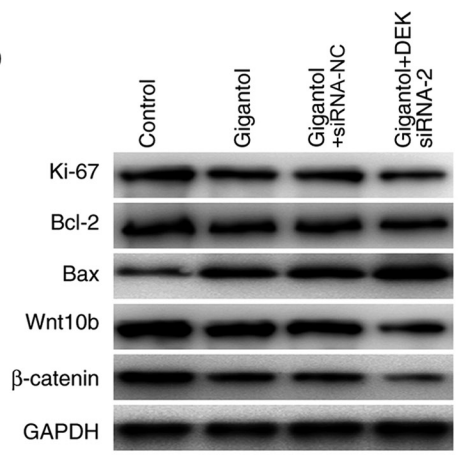

$\mathrm{E}$

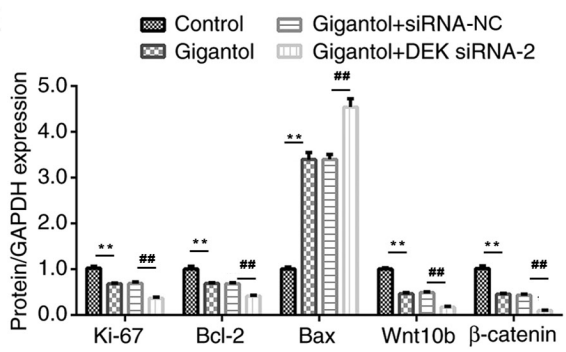

Figure 6. Gigantol decreases Ki-67 and Bcl-2 expression, increases Bax expression and inactivates the Wnt/ $\beta$-catenin signaling pathway. mRNA expression levels of (A) Ki-67, (B) Bcl-2 and (C) Bax were measured by reverse transcription-quantitative PCR. (D) The protein expression levels of Ki-67, Bcl-2, $\mathrm{Bax}, \mathrm{Wnt} 10 \mathrm{~b}$ and $\beta$-catenin were detected by western blotting and (E) semi-quantified. Data are presented as the mean $\pm \mathrm{SEM}$. ${ }^{* *} \mathrm{P}<0.01$ and ${ }^{\# \#} \mathrm{P}<0.01$. DEK, DEK proto-oncogene; siRNA, small interfering RNA. 
A

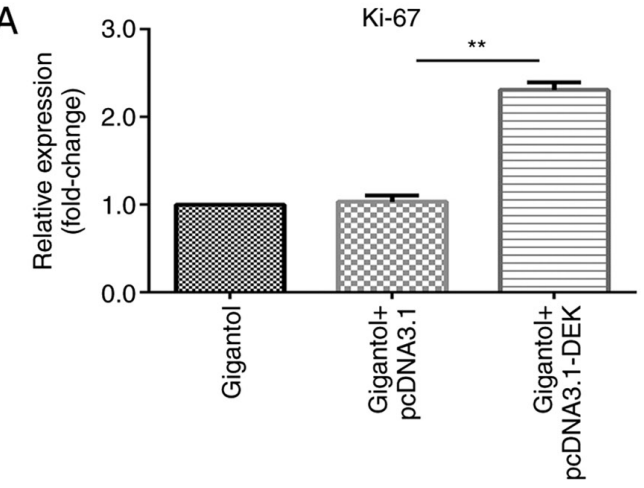

C

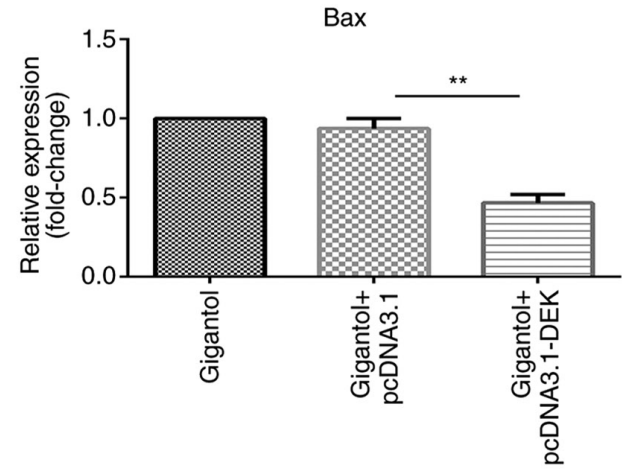

$\mathrm{B}$
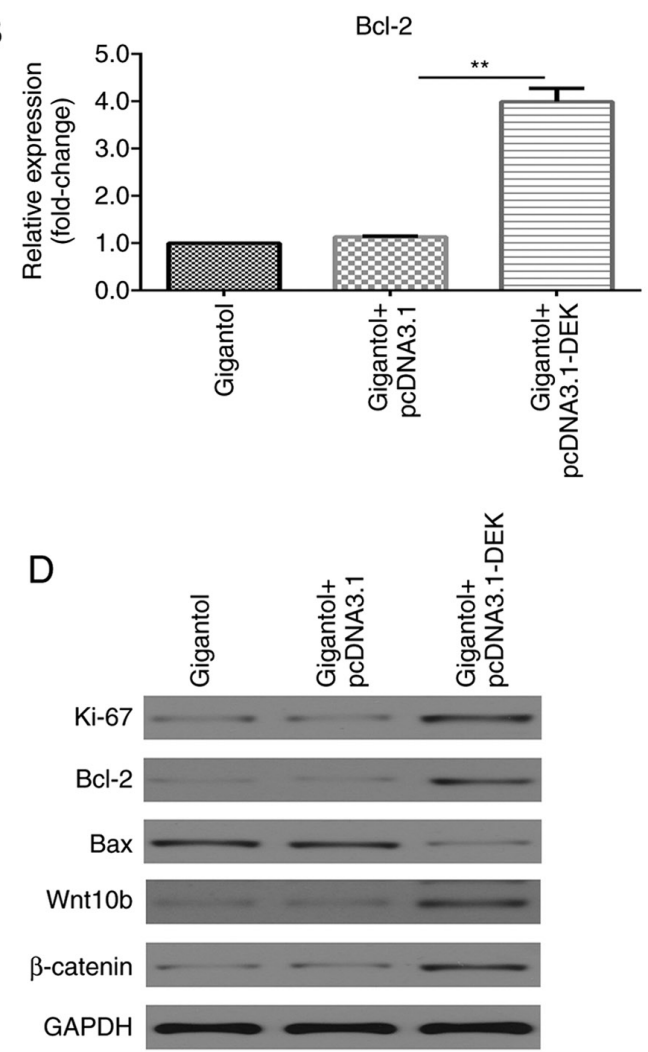

$\mathrm{E}$ Gigantol $\boxminus$ Gigantol+pcDNA3.1-DEK Gigantol+pcDNA3.1

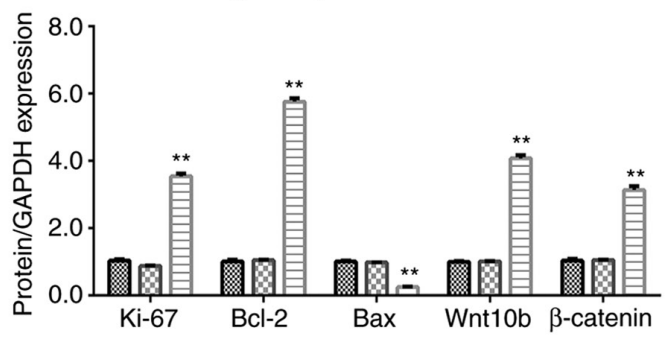

Figure 7. DEK overexpression reverses gigantol-mediated alterations to Ki-67, Bcl-2, Bax, Wnt10b and $\beta$-catenin expression levels. Reverse transcription-quantitative PCR was performed to measure the mRNA expression levels of (A) Ki-67, (B) Bcl-2 and (C) Bax. (D) Protein expression levels of Ki-67, Bcl-2, Bax, Wnt10b and $\beta$-catenin were determined by western blotting and (E) semi-quantified. Data are presented as the mean \pm SEM. ${ }^{* *} \mathrm{P}<0.01$ vs. gigantol + pcDNA3.1. DEK, DEK proto-oncogene.

Gigantol exhibits anticancer activities that regulate cellular processes in various types of human cancer (14-17). A number of studies have investigated the function of gigantol in lung cancer. For example, gigantol suppressed stem-like malignancy phenotypes of lung cancer cells, reducing anchorage-independent growth and the ability to form tumor spheroids (30). Additionally, gigantol inhibited EMT processes in H460 NSCLC cells $(16,31)$. Furthermore, gigantol suppressed NSCLC cell migration and invasion (17). In the present study, the effects of gigantol on NSCLC cell proliferation and apoptosis, and its underlying molecular mechanisms were assessed. The results showed that gigantol attenuated cell viability and decreased DEK expression levels in A549 cells compared with the control group. Additionally, DEK knockdown by siRNA enhanced gigantol-induced inhibition of proliferation, as well as gigantol-induced apoptosis. The results suggested that gigantol may inhibit proliferation and induced apoptosis by suppressing DEK expression in NSCLC.
$\mathrm{Ki}-67$ is present in proliferating cells and is therefore used to determine the proportion of proliferating cells in tumors (32). Bax is a proapoptotic factor of the Bcl-2 family, of which Bcl-2 is also an apoptosis-related factor (33). A previous study demonstrated that DEK knockdown downregulated $\mathrm{Bcl}-2$ expression and upregulated Bax expression (34). High DEK expression was also associated with increased Ki-67 expression (34). The Wnt/ $\beta$-catenin signaling pathway serves a pivotal role in NSCLC tumorigenesis, prognosis and resistance to therapy (35). A previous study reported that DEK promoted proliferation and $\beta$-catenin activity by regulating Wnt10b in breast cancer (36). In the present study, compared with the control group, gigantol reduced Ki-67, Bcl-2, Wnt10b and $\beta$-catenin expression levels, and increased Bax expression levels. Additionally, DEK siRNA-mediated knockdown enhanced these gigantol-induced effects. Collectively, the results indicated that gigantol suppressed decreased Ki-67 and Bcl-2 
expression levels, promoted increased Bax expression levels and inactivated the Wnt/ $\beta$-catenin signaling pathway possibly by regulating DEK.

The present study had a number of limitations. Firstly, only one NSCLC cell line was selected to explore the role of DEK. Secondly, the in vitro results were not verified in vivo. Therefore, further investigation is required.

In conclusion, the present study indicated that DEK was upregulated in NSCLC tissues and cells. Gigantol inhibited cell viability and decreased DEK expression levels. Furthermore, gigantol suppressed the proliferation and enhanced the apoptosis of NSCLC cells by decreasing Ki-67 and Bcl-2 expression, increasing Bax expression and inactivating the $\mathrm{Wnt} / \beta$-catenin signaling pathway, and this may be through DEK regulation. The results suggested that DEK knockdown may be associated with the anticancer activity of gigantol in NSCLC in vitro. Therefore, DEK may serve as a novel target to enhance the therapeutic effects of gigantol in patients with NSCLC.

\section{Acknowledgements}

Not applicable.

\section{Funding}

No funding was received.

\section{Availability of data and materials}

The datasets used and/or analyzed during the current study are available from the corresponding author on reasonable request.

\section{Authors' contributions}

YC and BR designed this study. YC, YH, HX and KC performed the experiments and the statistical analysis. $\mathrm{YH}$ and $\mathrm{HX}$ confirm the authenticatity of all the raw data. YC wrote and BR revised the manuscript. All authors read and approved the final manuscript.

\section{Ethics approval and consent to participate}

The present study was approved by the Ethics Committee of Baoji Traditional Chinese Medicine Hospital (Boaji, China; approval no. 201801001). Written informed consent was obtained from each participant.

\section{Patient consent for publication}

Not applicable.

\section{Competing interests}

The authors declare that they have no competing interests.

\section{References}

1. Carbone DP, Gandara DR, Antonia SJ, Zielinski C and Paz-Ares L: Non-small-cell lung cancer: Role of the immune system and potential for immunotherapy. J Thorac Oncol 10 974-984, 2015
2. Gu H, Chen J, Song Y and Shao H: Gastric adenocarcinoma predictive long intergenic non-coding RNA promotes tumor occurrence and progression in non-small cell lung cancer via regulation of the miR-661/eEF2K signaling pathway. Cell Physiol Biochem 51: 2136-2147, 2018.

3. Gridelli C, Rossi A, Carbone DP, Guarize J, Karachaliou N, Mok T, Petrella F, Spaggiari L and Rosell R: Non-small-cell lung cancer. Nat Rev Dis Primers 1:15009, 2015.

4. Kumarakulasinghe NB, van Zanwijk N and Soo RA: Molecular targeted therapy in the treatment of advanced stage non-small cell lung cancer (NSCLC). Respirology 20: 370-378, 2015.

5. Lam Y, Ng TB, Yao RM, Shi J, Xu K, Sze SC and Zhang KY: Evaluation of chemical constituents and important mechanism of pharmacological biology in Dendrobium plants. Evid Based Compement Alternat Med 2015: 841752, 2015.

6. Chen X, Wang F, Wang Y, Li X, Wang A, Wang C and Guo S Discrimination of the rare medicinal plant Dendrobium officinale based on naringenin, bibenzyl, and polysaccharides. Sci China Life Sci 55: 1092-1099, 2012.

7. Zheng S, Zhu Y, Jiao C, Shi M, Wei L, Zhou Y, Jin Q and Cai Y: Extraction and analysis of gigantol from Dendrobium officinale with response surface methodology. Molecules 23: $818,2018$.

8. Ng TB, Liu J, Wong JH, Ye X, Wing Sze SC, Tong Y and Zhang KY: Review of research on Dendrobium, a prized folk medicine. Appl Microbiol Biotechnol 93: 1795-1803, 2012.

9. Won JH, Kim JY, Yun KJ, Lee JH, Back NI, Chung HG Chung SA, Jeong TS, Choi MS and Lee KT: Gigantol isolated from the whole plants of Cymbidium goeringii inhibits the LPS-induced iNOS and COX-2 expression via NF-kappaB inactivation in RAW 264.7 macrophages cells. Planta Med 72: 1181-1187, 2006.

10. Simmler C, Antheaume $C$ and Lobstein A: Antioxidant biomarkers from Vanda coerulea stems reduce irradiated $\mathrm{HaCaT}$ PGE-2 production as a result of COX-2 inhibition. PLoS One 5: e13713, 2010

11. Fan C, Wang W, Wang Y, Qin G and Zhao W: Chemical constituents from Dendrobium densiflorum. Phytochemistry 57: 1255-1258, 2001

12. Wu J, Li X, Wan W, Yang Q, Ma W, Chen D, Hu J, Chen CO and Wei X: Gigantol from Dendrobium chrysotoxum Lindl. binds and inhibits aldose reductase gene to exert its anti-cataract activity: An in vitro mechanistic study. J Ethnopharmacol 198: 255-261, 2017.

13. Wu J, Li X, Fang H, Yi Y, Chen D, Long Y, Gao X, Wei X and Chen CY: Investigation of synergistic mechanism and identification of interaction site of aldose reductase with the combination of gigantol and syringic acid for prevention of diabetic cataract. BMC Complement Altern Med 16: 286, 2016.

14. Yu S, Wang Z, Su Z, Song J, Zhou L, Sun Q, Liu S, Li S, Li Y,Wang M, et al: Gigantol inhibits Wnt $/ \beta$-catenin signaling and exhibits anticancer activity in breast cancer cells. BMC Complement Altern Med 18: 59, 2018.

15. Chen H, Huang Y, Huang J, Lin L and Wei G: Gigantol attenuates the proliferation of human liver cancer HepG2 cells through the PI3K/Akt/NF- $\kappa B$ signaling pathway. Oncol Rep 37: 865-870, 2017.

16. Unahabhokha T, Chanvorachote $\mathrm{P}$ and Pongrakhananon V: The attenuation of epithelial to mesenchymal transition and induction of anoikis by gigantol in human lung cancer $\mathrm{H} 460$ cells. Tumour Biol 37: 8633-8641, 2016.

17. Charoenrungruang S, Chanvorachote P, Sritularak B and Pongrakhananon V: Gigantol, a bibenzyl from Dendrobium draconis, inhibits the migratory behavior of non-small cell lung cancer cells. J Nat Prod 77: 1359-1366, 2014.

18. von Lindern M, Breems D, van Baal S, Adriaansen $H$ and Grosveld G: Characterization of the translocation breakpoint sequences of two DEK-CAN fusion genes present in $\mathrm{t}(6 ; 9)$ acute myeloid leukemia and a SET-CAN fusion gene found in a case of acute undifferentiated leukemia. Genes Chromosomes Cancer 5: 227-234, 1992.

19. Waldmann T, Scholten I, Kappes F, Hu HG and Knippers R: The DEK protein-an abundant and ubiquitous constituent of mammalian chromatin. Gene 343: 1-9, 2004.

20. Hu HG, Scholten I, Gruss C and Knippers R: The distribution of the DEK protein in mammalian chromatin. Biochem Biophys Res Commun 358: 1008-1014, 2007.

21. Riveiro-Falkenbach E and Soengas MS: Control of tumorigenesis and chemoresistance by the DEK oncogene. Clin Cancer Res 16: 2932-2938, 2010 
22. Liu X, Qi D, Qi J, Mao Z, Li X, Zhang J, Li J and Gao W: Significance of DEK overexpression for the prognostic evaluation of non-small cell lung carcinoma. Oncol Rep 35: 155-162, 2016.

23. Livak KJ and Schmittgen TD: Analysis of relative gene expression data using real-time quantitative PCR and the 2(-Delta DeltaC(T)) method. Methods 25: 402-408, 2001.

24. Feng T,Liu Y,Li C, Li Z and Cai H: DEK proto-oncogene is highly expressed in astrocytic tumors and regulates glioblastoma cel proliferation and apoptosis. Tumour Biol 39: 1010428317716248, 2017.

25. Wise-Draper TM, Mintz-Cole RA, Morris TA, Simpson DS, Wikenheiser-Brokamp KA, Currier MA, Cripe TP, Grosveld GC and Wells SI: Overexpression of the cellular DEK protein promotes epithelial transformation in vitro and in vivo. Cancer Res 69: 1792-1799, 2009.

26. Hui W, Ma X, Zan Y, Song L, Zhang S and Dong L: MicroRNA-1292-5p inhibits cell growth, migration and invasion of gastric carcinoma by targeting DEK. Am J Cancer Res 8: $1228-1238,2018$

27. Piao J, Shang Y, Liu S, Piao Y, Cui X, Li Y and Lin Z: High expression of DEK predicts poor prognosis of gastric adenocarcinoma. Diagn Pathol 9: 67, 2014.

28. Lin L, Piao J, Gao W, Piao Y, Jin G, Ma Y, Li J and Lin Z: DEK over expression as an independent biomarker for poor prognosis in colorectal cancer. BMC Cancer 13: 366, 2013.

29. Zhou QC, Deng XF, Yang J, Jiang H, Qiao MX, Liu HH, Qian Z, Hou LL and Hu HG: Oncogene DEK is highly expressed in lung cancerous tissues and positively regulates cell proliferation as well as invasion. Oncol Lett 15: 8573-8581, 2018.
30. Bhummaphan N and Chanvorachote P: Gigantol suppresses cancer stem cell-like phenotypes in lung cancer cells. Evid Based Complement Alternat Med 2015: 836564, 2015.

31. Unahabhokha T, Chanvorachote P, Sritularak B, Kitsongsermthon J and Pongrakhananon V: Gigantol inhibits epithelial to mesenchymal process in human lung cancer cells. Evid Based Complement Alternat Med 2016: 4561674, 2016.

32. Gerdes J, Schwab U, Lemke H and Stein H: Production of a mouse monoclonal antibody reactive with a human nuclear antigen associated with cell proliferation. Int J Cancer 31: 13-20, 1983.

33. Harris MH and Thompson CB: The role of the Bcl-2 family in the regulation of outer mitochondrial membrane permeability. Cell Death Differ 7: 1182-1191, 2000.

34. Lin L, Piao J, Ma Y, Jin T, Quan C, Kong J, Li Y and Lin Z: Mechanisms underlying cancer growth and apoptosis by DEK overexpression in colorectal cancer. PLoS One 9: e111260, 2014.

35. Stewart DJ: Wnt signaling pathway in non-small cell lung cancer. J Natl Cancer Inst 106: djt356, 2014

36. Privette Vinnedge LM, Benight NM, Wagh PK, Pease NA, Nashu MA, Serrano-Lopez J, Adams AK, Cancelas JA, Waltz SE and Wells SI: The DEK oncogene promotes cellular proliferation through paracrine Wnt signaling in Ron receptor-positive breast cancers. Oncogene 34: 2325-2336, 2015.

(C) $\Theta$ This work is licensed under a Creative Commons Attribution-NonCommercial-NoDerivatives 4.0 International (CC BY-NC-ND 4.0) License. 